07

\title{
Структура деформируемого гетерогенного материала по данным акустической эмиссии и рентгеновской микротомографии
}

\author{
(C) Е.Е. Дамаскинская ${ }^{1}$, И.А. Пантелеев ${ }^{2}$, Д.Р. Гафурова ${ }^{3}$, Д.И. Фролов ${ }^{1}$ \\ ${ }^{1}$ Физико-технический институт им. А.Ф. Иофрфе РАН, \\ Санкт-Петербург, Россия \\ ${ }^{2}$ Институт механики сплошных сред УрО РАН, \\ Пермь, Россия \\ ${ }^{3}$ Московский государственный университет им. М.В. Ломоносова, \\ Москва, Россия \\ E-mail: Kat.Dama@mail.ioffe.ru;
}

(Поступила в Редакцию 22 января 2018 г.

В окончательной редакции 1 февраля 2018 г.)

\begin{abstract}
Проведены исследования накопления дефектов на разных этапах деформирования гетерогенного материала(гранита) с помощью двух неразрушающих методов: акустической эмиссии (AЕ) и рентгеновской компьютерной микротомографии (СТ). Квазистатические испытания цилиндрических образцов гранита Westerly проводились в условиях одноосного сжатия. Контроль за дефектообразованием осуществлялся с помощью мониторинга акустической эмиссии в режиме real-time. Для каждого из исследованных образцов было проведено несколько этапов нагружения и томографической съемки. Установлено, что анализ функционального вида (степенной или экспоненциальный) энергетических распределений АЕ-сигналов позволяет выделить область образца, в которой система дефектов перешла в состояние самоорганизованной критичности и образовались крупные трещины. Этот результат согласуется с данными рентгеновской томографии.
\end{abstract}

Работа выполнена при финансовой поддержке Российского Фонда Фундаментальных Исследований (гранты 16-05-00237, 17-05-00720).

DOI: 10.21883/FTT.2018.07.46122.017

\section{1. Введение}

Обширные исследования в области механики и физики прочности гетерогенных хрупких материалов, проведенные за последние 30-40 лет, привели к значительному прогрессу в понимании закономерностей накопления дефектов и образования очага разрушения. Было обнаружено, что накопление дефектов, возникающих в результате деформирования таких гетерогенных материалов, как горные породы, керамика и различные композиционные материалы, характеризуется наличием нескольких стадий [1-5]. Вначале нагружения происходит образование дефектов, распределенных случайным образом по всему объему деформируемого материала. Затем наблюдается локализация дефектообразования в определенной пространственной области, являющейся зоной формирования очага разрушения.

Для прогнозирования развития процесса разрушения и оценки степени критичности состояния деформированных материалов необходимо понять физические причины, вызывающие качественный переход от стадии дисперсного (стабильного) накопления повреждений к критической (или опасной) стадии, и найти критерий этого перехода.

Однако, локализация дефектов в некоторой области происходит на фоне дисперсного образования дефектов во всем объеме. В связи с этим, основной трудностью, возникающей при поиске критерия состояния материала, является отсутствие ярко выраженного перехода от одной стадии к другой.

В настоящее время исследование процесса накопления дефектов, осуществляются различными деструктивными и неразрушающими методами, такими как ультразвуковая техника, акустическая и электромагнитная эмиссия, фрактолюминесценция, нейтронная эмиссия, оптическая и электронная микроскопия и т.д. [6-17].

Ряд исследований посвящены поиску критериев перехода от стадии накопления случайных дефектов к стадии формирования очага разрушения $[3,4,9,10,15,18,19,20]$, но физические механизмы этого перехода и факторы, влияющие на него, по-прежнему остаются неясными. Так, ранее в теоретических работах было показано [5,19,20-22], что переход от одной стадии к другой происходит, когда плотность дефектов (трещин) в деформированном материале достигает критического значения, выше которого дефекты начинают взаимодействовать друг с другом своими локальными полями упругих напряжений. Однако, в эксперименте (лабораторном или натурном) невозможно корректно определить критическую плотность дефектов, выше которой происходит переход на „опасную“ стадию.

Другой подход к определению качественного перехода процесса накопления дефектов с первого на второй этап основан на концепции самоорганизованной критичности [23], в которой совокупность дефектов опи- 
сывается в терминах „простая-сложная система“ $[23,24]$. В [24,25] показано, что „простые системы“ характеризуются экспоненциальным распределением их параметров. В общем случае любая „простая система“ в результате эволюции становится „сложной“ ${ }^{\text {„с }}$ [24,25]. Это означает, что такая система не может быть описана как сумма невзаимодействующих „простых систем“, а ее свойства отличаются от свойств, „простых систем“. В процессе развития „сложная система““ переходит в состояние самоорганизованной критичности [25], характеризующееся степенными распределениями параметров [23].

Ранее проведенный нами анализ результатов лабораторных экспериментов по деформированию образцов гранита с использованием подхода „простая-сложная система“ выявил стадии, на которых процесс накопления дефектов развивается принципиально разным образом и которые характеризуются различными типами функции распределения энергии сигналов АЕ [26]. Было установлено, что на ранних этапах деформирования, когда наблюдается накопление дефектов, распределенных случайно по объему, распределение числа сигналов АЕ по энергии аппроксимируется экспоненциальной функцией. Согласно концепции самоорганизованной критичности $[23,24]$ совокупность дефектов, которые появляются на ранних этапах деформирования, можно рассматривать как „простую систему“. В такой системе практически отсутствует взаимодействие между дефектами.

По мере деформирования происходит локализация дефектообразования, которому соответствует степенное распределение числа сигналов АЕ по энергии. На этом этапе совокупность дефектов можно рассматривать как „сложную систему“, в которой проявляется взаимодействие между дефектами. „Сложные системы“ переходят в состояние самоорганизованной критичности [23], для которого характерно степенное распределение параметров системы.

Данная работа посвящена результатам прямой проверки высказанного ранее [26] предположения о механизме смены функционального вида энергетического распределения сигналов АЕ. Проведены исследования накопления дефектов на разных этапах деформирования с помощью двух неразрушающих методов: акустической эмиссии и рентгеновской компьютерной микротомографии.

\section{2. Методика экспериментов}

Исследование дефектной структуры образцов горных пород методом рентгеновской микротомографи до и после механических испытаний проводилось с помощью томографа ScyScan 1172 (Bruker, Belgium).

Особенностью метода рентгеновской компьютерной микротомографии является то, что пространственное разрешение коррелирует с толщиной образца [27].

Поскольку целью экспериментов является исследование закономерностей образования и накопления дефектов в объеме образца горной породы, размеры образца

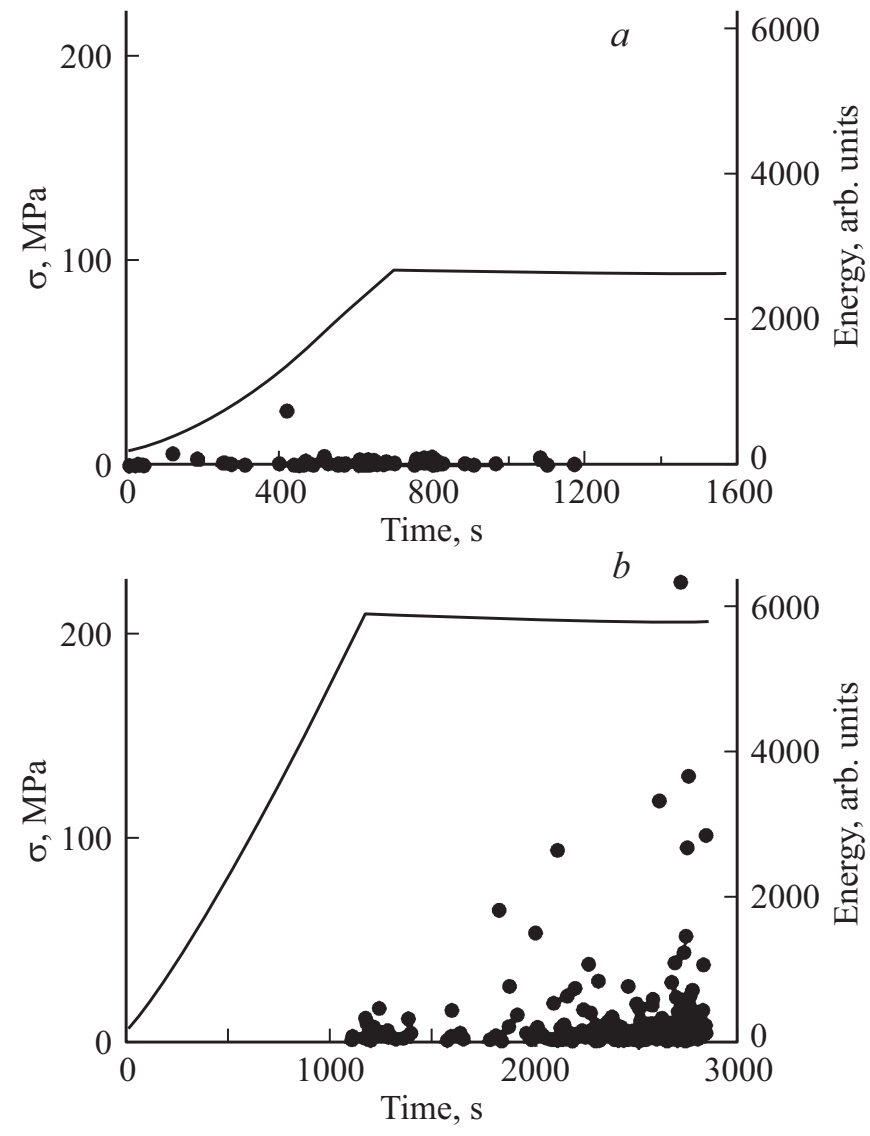

Рис. 1. Изменение напряжения (сплошная линия) и энергии отдельных сигналов $\mathrm{AE}$ (точки) на различных этапах деформирования образца: $a-$ первый этап, $b-$ заключительный этап.

должны быть достаточны для обеспечения возможности деформирования и получения достоверных результатов локации сигналов АЕ. Таким образом, была определена форма и размеры образцов, которые оптимальны как для механических испытаний, так и для томографии - это цилиндры диаметром $10 \mathrm{~mm}$ и высотой $20 \mathrm{~mm}$. Эксперименты проводились на образцах гранита Westerly, в котором средний размер зерна составляет $0.075 \mathrm{~mm}$ [28]. Пространственное разрешение томографических изображений при такой геометрии образца составило $\sim 3 \mu \mathrm{m}$. С учетом физических принципов томографии, конструктивных особенностей рентгеновской трубки и камеры томографа, достигнутое разрешение является максимально возможным для образцов данного размера [27].

Проведена серия испытаний (11 образцов). Томографическая съемка всех образцов с пространственным разрешением $3 \mu \mathrm{m}$, проведенная до начала механических испытаний, показала, что в материале не обнаружено дефектов типа трещин и пор.

Квазистатические испытания образцов гранита Westerly проводились в условиях одноосного сжатия на электромеханической испытательной машине Shimadzu AGX-Plus. Для мониторинга акустической эмиссии в режиме real-time в процессе деформирования был 
использован аппаратурный комплекс Amsy-5 Vallen System. Два широкополосных пьезопреобразователя акустической эмиссии AE105A с полосой 450-1150 kHz крепились к торцам образца, что позволило осуществить линейную локацию источников сигналов АЕ. В процессе эксперимента формировалась база данных, в которой записаны параметры отдельных сигналов АЕ - время излучения, координата источника и энергия. Точность определения координат источников сигналов АЕ $\sim 2 \mathrm{~mm}$.

Образец гранита деформировался поэтапно. На каждом этапе образец плавно (со скоростью $\sim 5 \mathrm{~N} / \mathrm{s}$ ) нагружался до определенной нагрузки $F$ и затем выдерживался при данном уровне нагрузки $F$ до тех пор, пока активность сигналов $\mathrm{AE}$ не спадала до нуля (рис. $1, a)$. На следующем этапе нагрузку $F$ увеличивали на $0.08 F_{\max }\left(F_{\max }-\right.$ разрушающая сила). Эксперимент был остановлен, когда на очередном этапе нагружения началось лавинообразное увеличение активности акустической эмиссии, которое указывает на приближающееся разрушение образца (рис. $1, b)$. В результате образец не разделился на отдельные части, что позволило провести заключительную томографическую съемку.

После каждого этапа образец разгружали, помещали в камеру томографа и выполняли томографическую съемку. Всего проведено 11 этапов нагружения и томографической съемки.

\section{3. Результаты экспериментов и обсуждение}

После проведения каждой томографической съемки образца формируется массив срезов (графических изображений), состоящий из $\sim 6000$ файлов. (Срез сечение, перпендикулярное оси цилиндрического образца.) Расстояние между срезами соответствует пространственному разрешению $(3 \mu \mathrm{m})$. На этих изображениях различной плотности материала соответствуют градации серого цвета. Дефектам типа трещин или пор соответствует черный цвет.

Был проведен анализ всего массива срезов и вычислена объемная доля дефектов после каждого этапа нагружения. Для этого с помощью программного пакета CTan выделялась узкая область шкалы серого цвета изображений вблизи черного и определялась объемная доля этой части спектра. Видно (рис. 2), что объемная доля дефектов растет по мере увеличения нагрузки. Это указывает на то, что в материале увеличивается доля несплошности.

Сопоставим результаты томографии с данными акустической эмиссии. На первых 9 этапах нагружения, т. е. при нагрузках меньших 0.9 от разрушающей, регистрировалось по несколько десятков сигналов АЕ. На последних двух этапах число сигналов увеличилось на два порядка. Детально остановимся на анализе акустоэмиссионных данных, полученных на последнем этапе нагружения. На распределении числа сигналов АЕ

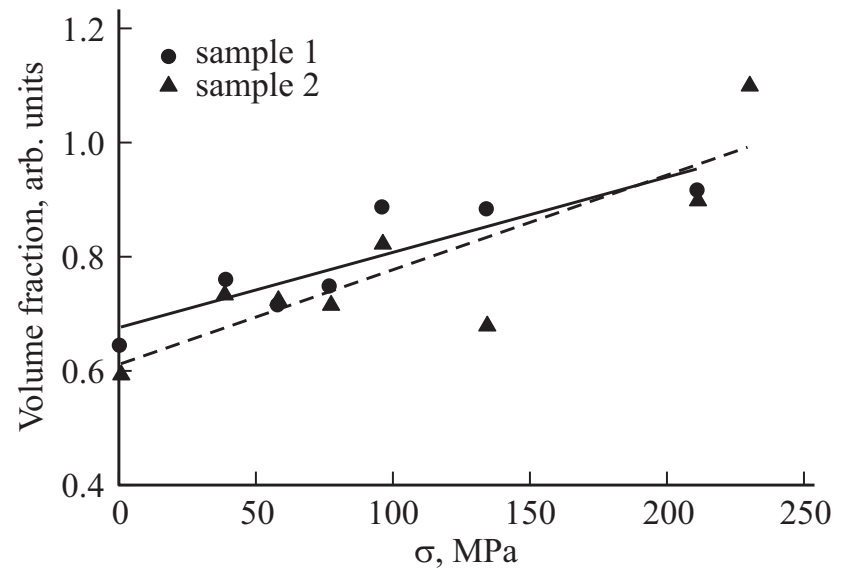

Рис. 2. Рентгеновская компьютерная микротомография. Зависимость объемной доли дефектов в образце от приложенного напряжения (для двух произвольно выбранных образцов).

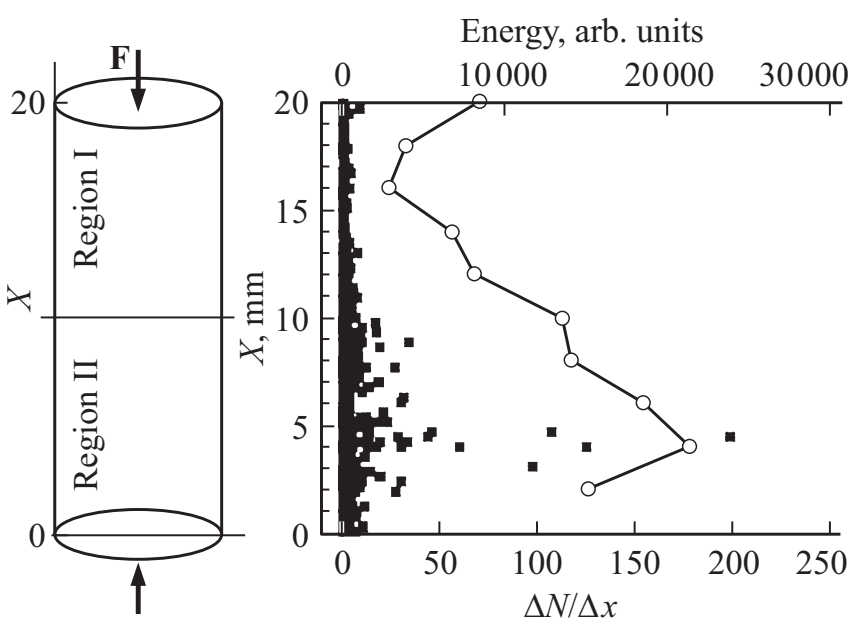

Рис. 3. Акустическая эмиссия (завершающий этап нагружения). Распределение числа сигналов АЕ по координате (линия) и энергия сигналов АЕ ( квадраты).

по координате, т. е. по высоте образца, (рис. 3, кривая 1) явно выделяется область, в которой генерируется большее, по сравнению со всем объемом образца, число сигналов АЕ. В этой же области генерируются сигналы с наиболее высокой энергией (рис. 3, квадраты).

Был проведен анализ распределений числа сигналов АЕ по энергии. На рис. 4 показано энергетическое распределение сигналов, зарегистрированных в верхней половине образца (Region I). Энергетические распределения этих сигналов AE могут быть аппроксимированы степенной или экспоненциальной функцией с почти равными коэффициентами детерминации $R^{2}$. Это иллюстрирует рис. 4 , на котором данное распределение построено в двойных логарифмических (рис. 4, $a$ ) и полулогарифмических (рис. 4,b) координатах. (Подобную картину мы наблюдали и в других экспериментах [26]). Согласно нашей гипотезе, этот результат означает, что в 

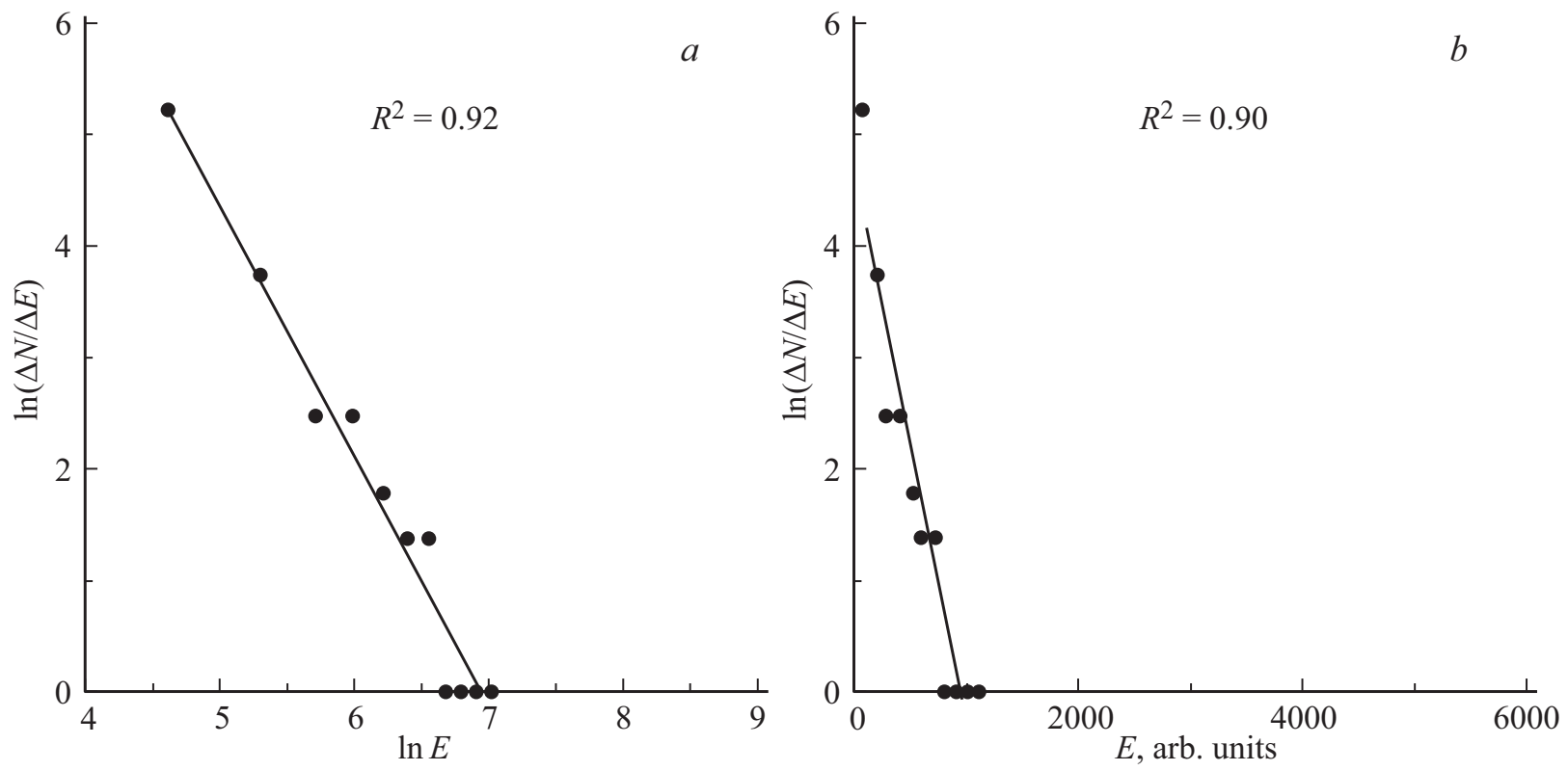

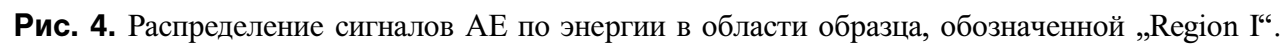
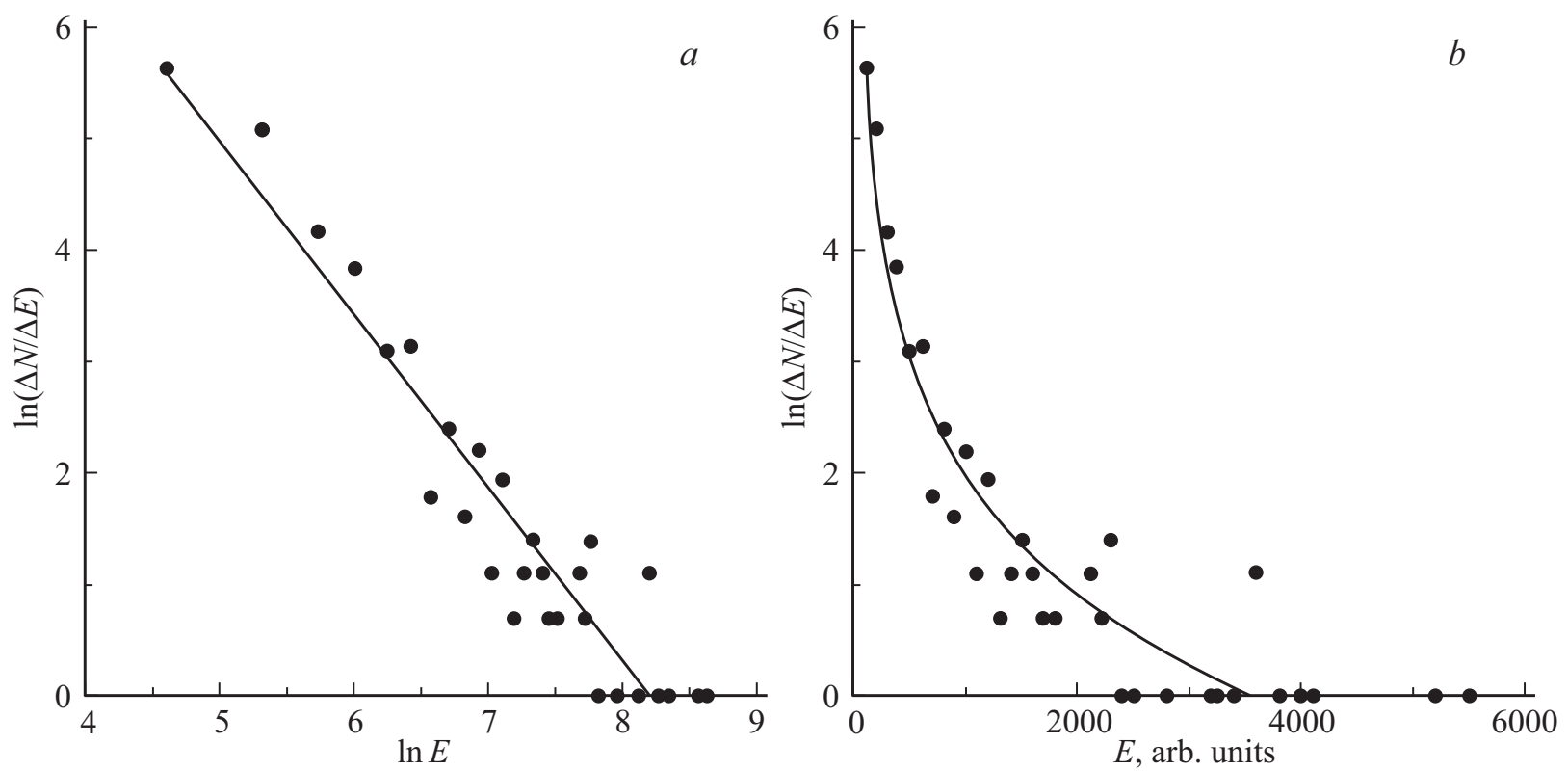

Рис. 5. Распределение сигналов АЕ по энергии в области образца, обозначенной „Region II“.

данной области материал образца находится на неопасной стадии накопления дефектов.

На рис. 5 показано энергетическое распределение сигналов, зарегистрированных в нижней половине образца (Region II). Явно видно, что данные аппроксимируются прямой линией в двойных логарифмических координатах. Следовательно, распределение степенное, которое указывает на то, что в данной области образца система дефектов перешла в состояние самоорганизованной критичности. То есть, в этой области материал образца находится на критической стадии процесса разрушения. Согласно концепции [23] дальнейшее развитие процесса приведет к потере целостности образца (разделению на части).

Таким образом, по данным акустической эмиссии можно выделить область образца, в которой совокупность дефектов (согласно нашей гипотезе) перешла в состояние самоорганизованной критичности, т.е. материал находится в опасном состоянии.

Было проведено сопоставление этого результата с данными рентгеновской томографии. Для этого вычислялась объемная доля дефектов в образце после последнего нагружения, но не для всего объема, а в отдельных слоях (рис. 6, $a$, кривая 1). Видно, что объемная доля 


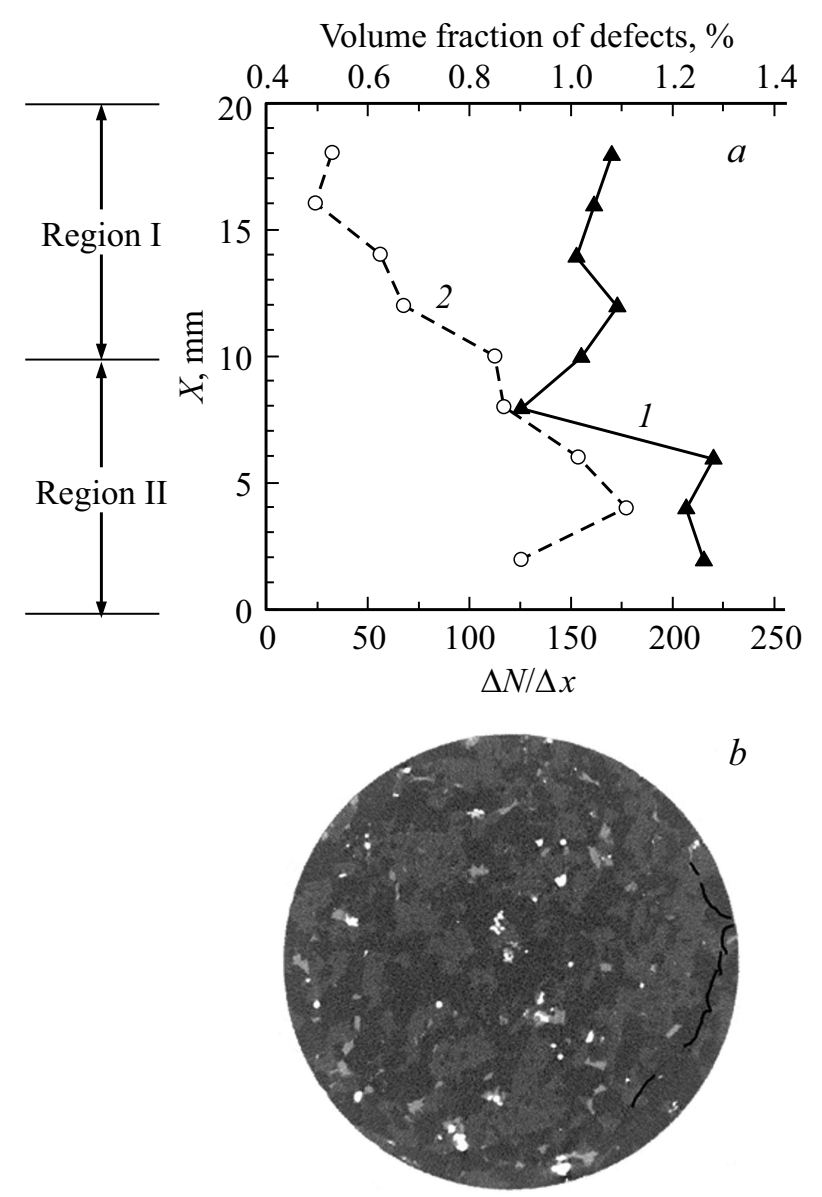

Рис. 6. Последняя стадия деформирования. Распределение объемной доли дефектов (кривая 1) и числа сигналов $\mathrm{AE}$ (кривая 2) вдоль высоты образца $(a)$; пример томографического среза $(b)$, на котором черная линия - образовавшаяся трещина.

дефектов значительно выше в нижней части образца (Region II). В этой же области наблюдается наибольшая концентрация источников $\mathrm{AE}$ (рис. 6, $a$, кривая 2). Как было показано выше (рис. 5) энергетическое распределение сигналов AE, зарегистрированных в Region II, описывается степенной функцией. На томографических срезах из этой области видны трещины (рис. 6, b). (Отметим, что на предыдущих этапах нагружения в этой области трещин не было обнаружено.)

\section{4. Заключение}

Таким образом, с помощью прямого сопоставления подтверждена гипотеза авторов о механизме смены функциональной формы распределения сигналов АЕ по энергиям, а сама форма распределения АЕ может использоваться как индикатор текущего состояния деформированного материала и критерий перехода к критическому этапу разрушения.
Экспоненциальный вид энергетического распределения сигналов AE указывает на некритическое (стабильное) состояние деформированного материала.

Степенной вид распределения указывает на то, что процесс накопления дефектов перешел на критическую („опасную“) стадию.

\section{Список литературы}

[1] D.A. Lockner, J.D. Byerlee, V. Kuksenko, A. Ponomarev, A. Sidorin. In Fault Mechanics and Transport Properties of Rocks / Ed. B. Evansand, T.-F. Wong. L. Academic Press (1992). P. 3.

[2] M. Petružálek, J. Vilhelm, V. Rudajev, T. Lokajívcek, T. Svitek. Int. J. Rock Mech. Mining Sci. 60, 208 (2013).

[3] Y. Ben-Zion, V. Lyakhovsky. Pure Appl. Geophys. 159, 2385 (2002).

[4] Y. Hamie, O. Katz, V. Lyakhovsky, Z. Reches, Yu. Fialko. Geophys. J. Int. 167, 1005 (2006).

[5] V. Kuksenko, N. Tomilin, E. Damaskinskaya, D. Lockner. Pure Appl. Geophys. 146, 2, 253 (1996).

[6] S. Peng, A.M. Johnson. Int. J. Rock Mech. Mining Sci. Geomech. Abs. 9, 37 (1972).

[7] A.G. Vostretsov, G.I. Kulakov, Yu.A. Timonenkov, G.E. Yakovitskaya. J. Mining Sci. 34, 4, 296 (1998).

[8] В.И. Веттегрень, В.С. Куксенко, И.П. Щербаков. ЖТФ. 83, 1, 144 (2013).

[9] A. Carpinteri, F. Cardone, G. Lacidogna. Experim. Mech. 50, 1235 (2010).

[10] A. Carpinteri, A. Chiodoni, A. Manuello, R. Sandrone. Strain. 47, 282 (2011).

[11] T.N. Dey, Wang Chi-Yuen. Int. J. Rock Mech. Mining Sci. 18, 199 (1981)

[12] R.L. Kranz. Int. J. Rock Mech. Mining Sci. 16, 37 (1979).

[13] Yoshizo Kawaguchi. Jpn. J. Appl. Phys. 37, 6A, 3495 (1998).

[14] В.Л. Гиляров, М.С. Варкентин, В.Е. Корсуков, М.М. Корсукова, В.С. Куксенко. ФТТ. 52, 7, 1311 (2010).

[15] A.V. Ponomarev, A.D. Zavyalov, V.B. Smirnov, D.A. Lockner. Tectonophys. 277, 57 (1997).

[16] T.H.W. Goebel, T.W. Becker, D. Schorlemmer, S. Stanchits, C. Sammis, E. Rybacki, G. Dresen. J. Geophys. Res. 117, B03310 (2012).

[17] Xinglin Lei, Shengli Ma. Earthq. Sci. 27, 6, 627 (2014).

[18] Л.Р. Ботвина. Физика Земли 10, 5 (2011).

[19] В.П. Тамуж, В.С. Куксенко. Микромеханика разрушения полимерных материалов. Зинатне, Рига (1978). 294 с.

[20] O.B. Naimark. Phys. Mesomech. J. 4, 4, 45 (2003).

[21] И.А. Пантелеев, О.А. Плехов, О.Б. Наймарк. Физика Земли 6, 43 (2012).

[22] О.Б. Наймарк. Письма в ЖЭТФ 67, 9, 751 (1998).

[23] P. Bak. How Nature Works: the Science of Self-Organized Criticality. Springer-Verlag (1996). 212 c.

[24] Г. Николис, И. Пригожин. Самоорганизация в неравновесных системах. Мир, М. (1979). 512 с.

[25] Г.Г. Малинецкий, А.Б. Потапов. Современные проблемы нелинейной динамики. Эдиториал УРСС, М. (2002). 360 с.

[26] E. Damaskinskaya, V. Hilarov, D. Frolov. AIP Conf. Proc., 1783, 020033 (2016).

[27] Teodor Tóth, Radovan Hudák. Acta Mech. Slovaca. 17, 4, 40 (2013).

[28] R.M. Stesky. Can. J. Earth. Sci. 15, 361 (1978).

Редактор Т.Н. Василевская 